Z. Klin. Chem. Klin. Biochem.

13. Jg. 1975, S. 361-366

\title{
Die Rolle von cyclischem AMP und Ca bei der Permeabilitätsänderung Mg-arm gewachsener Tumor- zellen
}

\author{
Von R. Averdunk, B. Ostapowicz und Th. Günther \\ Institut für Klinische Chemie und Klinische Biochemie und Zentralinstitut für Biochemie und Biophysik der Freien \\ Universität Berlin
}

(Eingegangen am 10. Februar/13. Mai 1975)

Zusammenfassung: Bei Mg-arm gewachsenen Yoshida-Ascites Tumorzellen ist die passive Permeabilität erhöht. Dies führt zu einem beschleunigten $\mathrm{Na}^{+}$-Efflux. Die Aufnahme von $\gamma$-Aminobuttersäure ist verringert. Ihre Abgabe, sowie die Aufnahme und Abgabe von $\alpha$-Methylglucopyranosid sind unverändert. Der Gehalt an Ado-3':5'P ist bei den Mgarm gewachsenen Zellen leicht erhöht. Zugabe von Dibutyryl-Ado-3': $5^{\prime} P$ führt zu ähnlichen, aber geringeren Veränderungen der zellulären Kationenkonzentrationen als der Mg-Mangel. Es wird diskutiert, ob die Auswirkungen des $\mathrm{Mg}$ Mangels durch Ado-3': $: 5^{\prime} P$ und/oder durch eine Erhöhung des Ca-Gehaltes in der Zelle ausgelöst werden können.

\section{The role of cyclic AMP and $C a$ in the altered permeability of tumour cells grown with a deficiency of $\mathrm{Mg}$}

Summary: Yoshida-Ascites tumour cells grown with a deficiency of $\mathrm{Mg}$ show an increased passive permeability. This results in an accelerated efflux of $\mathrm{Na}^{+}$. The uptake of aminobutyric acid is decreased, while its release is unchanged. The uptake and release of $\alpha$-methylglucopyranoside are unchanged. In the cells grown with a deficiency of $\mathrm{Mg}$, the concentration of cyclic AMP is slightly increased. The addition of dibutyryl cyclic AMP results in similar but smaller changes in the cellular concentration of cations, to those found in $\mathrm{Mg}$ deficiency. It is suggested that the effects of $\mathrm{Mg}$ deficiency result from the action of cyclic AMP and/or an increase in the $\mathrm{Ca}$ concentration of the cell.

\section{Einführung}

Im Mg-Mangel treten hauptsächlich Veränderungen des Elektrolytstoffwechsels auf $(1,2)$. Besonders ausgeprägt ist eine Abnahme des $\mathrm{K}^{+}$-Gehaltes und eine etwas geringere Zunahme des $\mathrm{Na}^{+}$-Gehaltes der Zellen. Die extrazellulären Konzentrationen von $\mathrm{Na}^{+}$und $\mathrm{K}^{+}$bleiben konstant (1). Im Tierversuch findet man bei Mg-Mangelernährung eine starke Abnahme der $\mathrm{Mg}$-Konzentration im Serum und eine geringe Abnahme des Mg-Gehaltes in den Zellen. Der Ca-Gehalt der Zellen steigt an $(1,3,4)$.

Die einzelnen Organe verhalten sich im Mg-Mangel unterschiedlich. Die stärksten Veränderungen wurden im braunen Fettgewebe der Ratte gefunden (3). Wahrscheinlich wird hier im Mg-Mangel durch die starke sympathische Innervation besonders viel Noradrenalin freigesetzt (4). Es erscheint daher möglich, daß die im Mg-Mangel auftretenden Veränderungen des Elektrolytstoffwechsels zum Teil auf einer Zunahme des Ado- $3^{\prime}: 5^{\prime} P$ -
Gehaltes der Zellen beruhen. Eine andere Möglichkeit wäre eine direkte Alteration der Zellmembran bzw. ihrer Permeabilität. Zur Klärung dieser Fragen untersuchten wir verschiedene Transportsysteme, sowie den Gehalt an Ado-3': $5^{\prime} P$ und $C a$ von Tumorzellen, die in einem Mg-armen Medium gezüchtet wurden, weil bei diesen Zellen eine Beeinflussung durch Catecholamine entfällt.

\section{Material und Methoden}

Yoshida-Ascites-Tumorzellen wurden 5 Tage in der Suspensionskultur in Eagle-Spinner-Medium, dem $10 \% \mathrm{Mg}$-frei dialysiertes Kälberserum und $5 \%$ fötales Kälberserum zugegeben wurde, gezüchtet (2). Die Mg-Konzentration betrug im Normalmedium $1,2 \mathrm{mmol} / \mathrm{l}$ und im $\mathrm{Mg}$-armen Medium 0,18 mmol/l.

Zur Bestimmung der abgestorbenen Zellen wurden $0,2 \mathrm{ml}$ Zellsuspension $\left(5 \times 10^{6}\right.$ Zellen $\left./ \mathrm{ml}\right)$ mit $0,1 \mathrm{ml}$ TrypanblauLösung $(10 \mathrm{~g} / 1$ in $0,15 \mathrm{~mol} / 1 \mathrm{NaCl})$ versetzt und 30 min danach der Antcil der blaugefärbten (d.h. abgestorbenen) Zellen zur 
Beurteilung der Kultur (2) ausgezählt. Zellzählungen wurden mit dem Coulter-Counter Modell B vorgenommen.

Die Proteinbestimmungen crfolgten mit der Biuretmethode nach Auflösen der Zellen in $0,1 \mathrm{~mol} / 1 \mathrm{NaOH}$ (5).

Zur Bestimmung des zellulären $\mathrm{Na}^{+}$- und $\mathrm{K}^{+}$-Gehaltes wurden die Zellen durch $10 \mathrm{~min}$ Zentrifugieren bei $70 \mathrm{~g}$ vom Kulturmedium abgetrennt und zweimal auf der Zentrifuge mit $0,1 \mathrm{~mol} / 1 \mathrm{MgCl}_{2}$ gewaschen. Die Zellen wurden dann mit $1 \mathrm{~kg} / 1$ Trichloressigsäure versetzt und anschließend 1:10 mit dest. Wasser verdünnt. Das ausgefallene Protein wurde abzentrifugiert und im Überstand $\mathrm{Na}^{+}$und $\mathrm{K}^{+}$flammenfotometrisch gemessen.

Zur Bestimmung des Mg- und Ca-Gehaltes wurden die Zellen in $0,15 \mathrm{~mol} / \mathrm{l} \mathrm{NaCl}$ gewaschen und in gleicher Weise mit Trichloressigsäure aufgeschlossen. Im Trichloressigsäure-Extrakt wurde $\mathrm{Mg}$ und $\mathrm{Ca}$ mit dem Atomabsorptionsspektrometer gemessen. Vergleichsuntersuchungen, bei denen die Zellen durch Phthalsäuredibutylester zentrifugiert wurden (6), führten zu gleichen Ergebnissen.

Zur Bestimmung der $\left[{ }^{3} \mathrm{H}\right.$ ]Ouabain-Bindung wurden die Zellen bis zum Erreichen eines Sättigungswertes bei $37^{\circ} \mathrm{C}$ und bei $4^{\circ} \mathrm{C}$ mit $100 \mathrm{nmol} / 1\left[^{3} \mathrm{H}\right]$ Ouabain inkubiert. Die Zellen wurden durch $30 \mathrm{~s}$ langes Zentrifugieren bei $10.000 \mathrm{U} / \mathrm{min}$ (Eppendorf-Mikroeinheit) vom Medium abgetrennt, dreimal mit eiskalter $0,15 \mathrm{~mol} / 1 \mathrm{NaCl}$ gewaschen, in $0,01 \mathrm{~mol} / 1 \mathrm{NaOH}$ aufgelöst, quantitativ in Szintillationsflüssigkeit aufgenommen und in einem Beckman-Szintillationsspek trometer gemessen. Die Anzahl der gebundenen Ouabain-Moleküle pro Zelle (OBS) wurde nach folgender Formel (Gleichung (1)) berechnet (6):

$$
\begin{aligned}
\text { OBS } & =\frac{\text { Imp. } / \mathrm{min} \cdot \text { Zelle } \times 100 / \mathrm{e} \times \mathrm{N}}{\mathrm{S} \times 2,2 \times 10^{12}} \\
\mathrm{e} & =\text { Zählausbeute in \% } \\
\mathrm{S} & =\text { spezifische Aktivität in Ci/mol } \\
\mathrm{N} & =\text { Loschmidt'sche Zahl. }
\end{aligned}
$$

Zur Bestimmung der unspezifischen Ouabain-Bindung wurden die Zellen $10 \mathrm{~min}$ mit $1 \mathrm{mmol} / \mathrm{l}$ unmarkiertem Ouabain vorinkubiert. Anschließend wurden $100 \mathrm{nmol} / 1\left[{ }^{3} \mathrm{H}\right]$ Ouabain zugesetzt. Nach einer weiteren Inkubationszeit von $60 \mathrm{~min}$ wurden die Zellen gewaschen, aufgeschlossen und die ${ }^{3} \mathrm{H}$-Aktivität bestimmt (s. o.). Die gefundene unspezifische ${ }^{3} \mathrm{H}$-Aktivität wird von den Werten subtrahiert, die in Gegenwart von $100 \mathrm{nmol} / 1$ $\left[{ }^{3} \mathrm{H}\right]$ Ouabain gefunden wurden.

Zur Bestimmung des $\mathrm{Na}^{+}$-Efflux wurden die Zellen bei $37^{\circ} \mathrm{C}$ mit $20 \mathrm{mCi}^{22} \mathrm{Na} / \mathrm{l}$ in Eagle-Medium, das $5 \%$ fötales Kälberserum enthielt, beladen. Nach 60 min wurden die Zellen zweimal mit radionuklidfreiem, gekühltem Eagle-Medium gewaschen und im gleichen Medium bei $37^{\circ} \mathrm{C}$ in einer Zelldichte von $1 \times 10^{7} \mathrm{Zellen} / \mathrm{ml}$ weiter inkubiert. Nach verschiedenen Zeiten wurden 0,5 ml der Zellsuspension entnommen, auf der Eppendorf-Zentrifuge bei $10.000 \mathrm{U} / \mathrm{min}$ zentrifugiert und die Zellen, sowie ein Aliquot des Überstandes im Packard-AutogammaZähler gemessen. Die Geschwindigkeitskonstante wurde nach Radkliffe (7) ermittelt:

$\begin{aligned} & \begin{array}{l}\text { Geschwindig- } \\ \text { keits- } \\ \text { konstante }\end{array} \\ & \text { knt }\end{aligned}=\ln \frac{\mathrm{Imp} \cdot / \mathrm{min} \cdot \mu \mathrm{l} \text { Zellwasser nach } 10 \mathrm{~min}}{\mathrm{Imp} / \mathrm{min} \cdot \mu \mathrm{l} \text { Zellwasser nach } 0 \mathrm{~min}} \times \mathrm{h}^{-1}$

Zur Bestimmung der Aufnahme von $\gamma$-Aminobuttersäure und $\alpha$-Methylglucopyranosid wurden die Zellen bei $37^{\circ} \mathrm{C}$ in einer Konzentration von $1-2 \times 10^{7}$ Zellen/ml in Krebs-Ringerphosphatlösung pH 7,0 und einer Osmolarität von $275 \mathrm{~m}$ osmol/1 nach Zugabe von $\left[{ }^{14} \mathrm{C}\right] \gamma$-Aminobuttersäure bzw. $\left[{ }^{14} \mathrm{C}\right] \alpha$-Methylglucopyranosid inkubiert und $z u$ verschiedenen Zeiten $1 \cdot \mathrm{ml}$ der Zellsuspension entnommen, in einer Eppendorf-Zentrifuge bei $10.000 \mathrm{U} / \mathrm{min} 30 \mathrm{~s}$ zentrifugiert, der Überstand dekantiert, das Gefäß ausgetupft und das Gewicht der Zellen bestimmt. Bei der Bestimmung der $\alpha$-Methylglucopyranosid-Aufnahme und Abgabe wurde dem Medium $1 \mathrm{mmol} / 1$ Natriumpyruvat zugesetzt. Die Zellen wurden mit $0,5 \mathrm{ml} 100 \mathrm{~g} / \mathrm{l}$ Trichloressigsäure aufge-
schlossen, das Protein bei $1.400 \mathrm{~g}$ bzentrifugiert essigsäure ausgeäthert und $0,1 \mathrm{ml}$ des Überstandes mit $10 \mathrm{ml}$ Szintillationsflüssigkeit versetzt. Die ${ }^{14} \mathrm{C}$-Aktivität wurde im Beckman-Szintillationsspektrophotometer LS 100 gemessen.

$\mathrm{Da}$ in dieser Versuchsanordnung die im Extrazellulärraum enthaltene ${ }^{14} \mathrm{C}$-Aktivität mitgemessen wird, dieser sich aber durch einen unterschiedlichen Anteil von abgestorbenen Zellen in den einzelnen Versuchen unterschied, wurde der Extrazellulärraum mit ${ }^{14} \mathrm{C}$-Inulin in jedem einzelnen Versuch bestimmit. Die Werte lagen zwischen 15 und $20 \%$ des Volumens des Zellsediments. Zur Bestimmung der Aufnahmegeschwindigkeit wurde die dem extrazellulären Raum entsprechende ${ }^{14} \mathrm{C}$-Aktivität der $\boldsymbol{\gamma}$-Aminobuttersäure bzw. des $\alpha$-Methylglucopyranosids subtrahiert.

Zur Bestimmung der Abgabe von $\gamma$-Aminobuttersäure und $\alpha$-Methylglucopyranosid wurden die Zellen unter gleichen Bedingungen wie bei den Aufnahme-Versuchen vorinkubiert. Nach Erreichen der Steady-State-Konzentration, was $60 \mathrm{~min}$ dauerte, wurden die Zellen zweimal gewaschen und in $\gamma$-Aminobuttersäure- sowie $\alpha$-Methylglucopyranosid-freiem Medium inkubiert. Nach verschiedenen Zeiten wurde $1 \mathrm{ml}$ Zellsuspension entnommen, zentrifugiert, der Überstand dekantiert und die ${ }^{14} \mathrm{C}$-Aktivität der Zellen in gleicher Weise gemessen, wie bei den Versuchen zur Aufnahme von $\alpha$-Methylglucopyranosid und $\gamma$-Aminobuttersäure. Die Werte wurden graphisch dargestellt. Aus dem linearen Teil der Kurve wurde unter Berücksichtigung der bekannten spez. Aktivität und Zählausbeute die Aufnahmebzw. Abgabegeschwindigkeit errechnet.

Der Gehalt an Ado-3': $5^{\prime} \boldsymbol{P}$ wurde nach Aufschluß der Zellen mit $0,1 \%$ Triton $X-100$ mit dem Test-Kit der $\mathrm{Fa}$. Boehringer, Mannheim, bestimmt. Wurden mit dieser Methode 10

Einzelbestimmungen aus der gleichen Zellsuspension vorgenommen, ergab sich ein VK von $4,12 \%$. In einer ZumischAnalyse wurde zu dem Extrakt von $1 \times 10^{8}$ Zellen, die für die Messung eingesetżt wurden, 1-8 $\mu$ g Ado-3':5'P zugesetzt. Es wurden durchschnittlich $96 \%$ der zugegebenen Ado-3': $5^{\prime} P$ Menge wiedergefunden. Wurden größere Mengen zugemischt, sank die Menge des wiedergefundenen Ado- $3^{\prime}: 5^{\prime} P$.

\section{Ergebnisse}

\section{$\mathrm{Na}^{+}$-Transport}

In früheren Versuchen (2) hatte sich gezeigt, daß bei Mg-arm gewachsenen Yoshida-Zellen der in trażelluläre $\mathrm{K}^{+}$-Gehalt $\mathrm{ab}$ - und der intrazelluläre $\mathrm{Na}^{+}$-Gehalt zugenommen hatten, der ${ }^{42} \mathrm{~K}$-Efflux war beschleunigt. Da $\mathrm{K}^{+}$- und $\mathrm{Na}^{+}$-Transport bei somatischen Zellen gekoppelt sind, bestimmten wir den ${ }^{22} \mathrm{Na}$-Efflux (Abb. 1). Errechnet man aus den halblogarithmischen Auftragungen der Gleichung (2) die Geschwindigkeitskonstanten des $\mathrm{Na}^{+}$Efflux, so ergibt sich für normal gewachsene Zellen eine Geschwindigkeitskonstante $\mathrm{k}=0,015 \times \mathrm{h}^{-1}$ und für die $\mathrm{Mg}$-armen Zellen $\mathrm{k}=0,020 \times \mathrm{h}^{-1}$. Dies zeigt, daß bei den Mg-arm gewachsenen Zellen der $\mathrm{Na}^{+}$-Efflux, d. h. der aktive $\mathrm{Na}^{+}-$Transport aus der Zelle, erhöht ist. Daraus ergibt sich die Frage, ob die Aktivität der $\mathrm{Na}^{+}-\mathrm{K}^{+}$. abhängigen ATPase auch erhöht ist. Eine solche veränderte ATPase-Aktivität konnte nicht nàchgewiesen werden (2). Allerdings wurde die Bestimmung der ATPase-Aktivität an aufgeschlossenen Zellen jeweils bei den gleichen optimalen $\mathrm{Na}^{+}-, \mathrm{K}^{+}$- und ATP-Konzentrationen durchgeführt und auf die Proteinmenge im Ansatz bezogen. Die Anżahl der $\mathrm{Na}^{+}$-, $\mathrm{K}^{+}$-Pumpstellen pro Zelle und die Aktivität der ATPase in der intakten Zelle können aber von der ATPase-Aktivität einer Membrạnpräparation verschieden sein. Deshalb haben wir die Ouabainbindung an intakten Zellen bestimmt. 


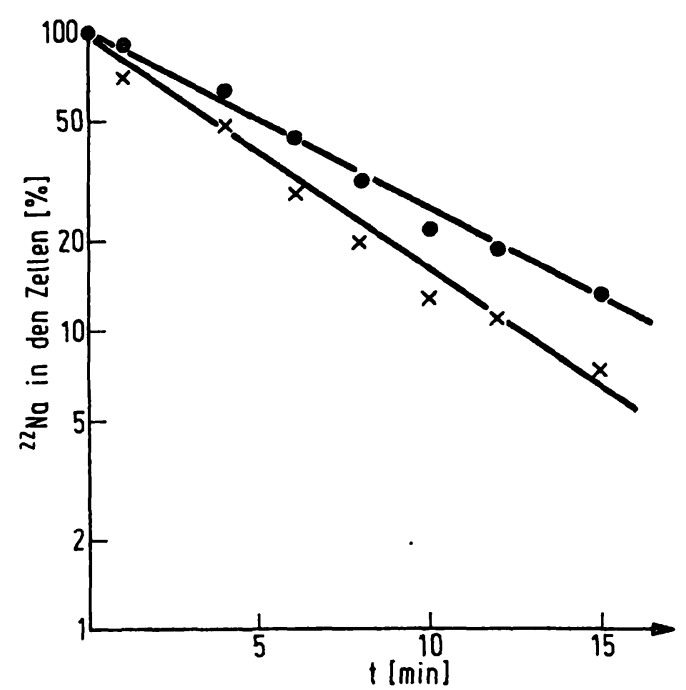

Abb. 1. Na-Efflux bei Mg-arm gewachsenen Yoshida-Tumorzellen Die Inkubation der mit ${ }^{22} \mathrm{Na}$ vorbeladenen Zellen wurde zu verschiedenen Zeiten unterbrochen und die ${ }^{22} \mathrm{Na-}$ Aktivität der Zellen bestimmt. Der Variationskoeffizient der Methode beträgt $2,96 \%$ bei $n=10$ Bestimmungen. - normale Zellen, $\mathrm{k}=0,015 \cdot \mathrm{h}^{-1}$ $\mathrm{x}$ - $\mathrm{x}$ Mg-arme Zellen, $\mathrm{k}=0,020 \cdot \mathrm{h}^{-1}$

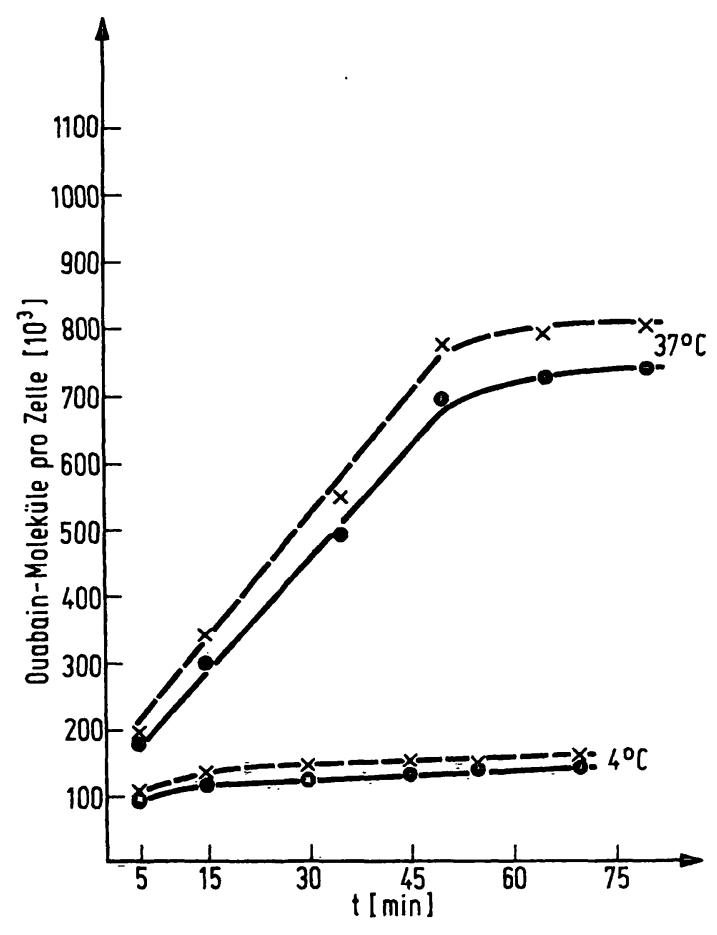

Abb. 2. Bindung von Ouabain an Yoshida-Tumorzellen $5 \times 10^{6}$ Zellen wurden in Gegenwart von $100 \mathrm{nmol} / \mathrm{l}$ $\left[{ }^{3} \mathrm{H}\right]$ Ouabain bei $37^{\circ} \mathrm{C}$ bzw. $4^{\circ} \mathrm{C}$ inkubiert. Nach verschiedenen Zeiten wurde die ${ }^{3} \mathrm{H}$-Aktivität der Zellen bestimmt. Die angegebenen Werte wurden nach Subtraktion der unspezifischen Bindung erhalten. Der Variationskoeffizient beträgt $3,81 \%$ bei $n=9$ Bestimmungen. - - normale Zellen x - x Mg-arme Zellen

\section{$\left[{ }^{3} \mathrm{H}\right] \mathrm{Ouabain}-\mathrm{Bindung}$}

Aus Abbildung 2 ist zu ersehen, daß Yoshida-AscitesTumorzellen bei $37^{\circ} \mathrm{C}$ etwa $8 \times 10^{5}$ Ouabain-Bindungsstellen pro Zelle besitzen. Bei $4^{\circ} \mathrm{C}$ wurden nur etwa $2 \times 10^{5}$ Ouabain-Moleküle pro Zelle gebunden. Mg-arm gewachsene Zellen binden $\left[{ }^{3} \mathrm{H}\right]$ Ouabain, wie aus den Steigungen der Bindungskurven zwischen 5 und $45 \mathrm{~min}$ hervorgeht, nicht schneller. Die Anzahl der OuabainBindungsstellen pro Zelle ist aber um $8 \%$ höher als bei den Kontrollzellen. Dieser geringe Unterschied ist bei dem dieser Methode anhaftenden Variationskoeffizienten von $\mathrm{VK}=4,37 \%(\mathrm{n}=10$ Einzelmessungen $)$ nicht signifikant, so daß geschlossen werden kann, daß die Zahl der $\mathrm{Na}^{+}$-, $\mathrm{K}^{+}$-Pumpstellen bei $\mathrm{Mg}$-arm gewachsenen Zellen sich nicht verändert hat.

\section{Aminosäure- und Glucosetransport}

Neben dem aktiven Transport von $\mathrm{Na}^{+}$und $\mathrm{K}^{+}$sind auch die Transportsysteme für Aminosäuren und Zucker für den Zellstoffwechsel wichtig. Zur Charakterisierung weiterer Membranfunktionen untersuchten wir, ob bei den Yoshida-Zellen durch den Mg-Mangel auch der Transport von Aminosäuren und Glucose betroffen ist. Veränderungen des Aminosäure- und Glucosetransportes könnten zusätzlich für die beschriebene Verringerung des Wachstums der Zellen (2) in der Mg-armen Kultur verantwortlich sein. Außerdem kann der Aminosäuretransport vom $\mathrm{Na}^{+}$-Gradienten abhängig sein (8). Unter Mg-Mangelbedingungen gewachsene Zellen haben um $18 \%$ weniger $\gamma$-Aminobuttersäure aufgenommen als die Kontrollzellen (Tab. 1). Die Abgabe von $\gamma$-Aminobuttersäure und die Aufnahme und Abgabe von $\alpha$-Methylglucopyranosid unterschieden sich nicht. (Tab. 1).

Tab. 1. Aufnahme und Abgabe von $\gamma$-Aminobuttersäure (ABS) und $\alpha$-Methylglucopyranosid (MGP) bei normalen und $\mathrm{Mg}$-arm gewachsenen Tumorzellen.

Angaben in $\mu \mathrm{mol} / \mathrm{min} \times 1$ Zellwasser

Die Aufnahmegeschwindigkeiten von $\gamma$-Aminobuttersäure oder $\alpha$-Methylglucopyranosid wurden bei ciner Konzentration von $0,5 \mathrm{mmol}$ und $1 \mathrm{mCi} / \mathrm{l}$ bestimmt. Die ${ }^{14} \mathrm{C}$-Aktivität in den Zellen wurde alle 5 min gemessen. Nach Auftragen der intrazellulären Konzentration von $\gamma$-Aminobuttersäure bzw. $\alpha$-Methylglucopyranosid gegen die Zeit wurde die Aufnahmegesch windigkeit aus der Steigung des linearen Anteils der Kurve ermittelt. Zur Bestimmung der Abgabegesch windigkeit wurden die Zellen mit $\left[{ }^{14} \mathrm{C}\right] \gamma$-Aminobuttersäure bzw. $\alpha$-Methylglucopyranosid vorbeladen und die nach Waschen und Reinkubation in den Zellen verbliebene ${ }^{14} \mathrm{C}$-Aktivität in 5 min-Abständen bestimmt. Die Berechnung erfolgte wie bei der Aufnahme.

\begin{tabular}{lll}
\hline & Kontrolle & Mg-arm \\
\hline ABS-Aufnahme & $1,87 \pm 0,15$ & $1,52 \pm 0,10$ \\
ABS-Abgabe & $1,01 \pm 0,15$ & $0,92 \pm 0,10$ \\
MGP-Aufnahme & $6,67 \pm 0,70$ & $5,19 \pm 0,62$ \\
MGP-A bgabe & $4,33 \pm 0,51$ & $3,78 \pm 1,63$ \\
\hline
\end{tabular}




\section{Ado- $3^{\prime}: 5^{\prime} P$ und $\mathrm{Ca}-\mathrm{Gehalt}$}

Ein vermehrter passiver $\mathrm{K}^{+}$-Efflux und $\mathrm{Ca}$-Influx wird in Leberzellen durch Noradrenalin bzw. Ado-3':5'P ausgelöst (9). Bei normalen Yoshida-Zellen beträgt der CaGehalt $1,47 \mathrm{mmol} / \mathrm{kg}$ Feuchtgewicht und der $\mathrm{Mg}$-Gehalt $15,7 \mathrm{mmol} / \mathrm{kg}$ Feuchtgewicht, während die Mg-arm gewachsenen Zellen einen Ca-Gehalt von $2,19 \mathrm{mmol} / \mathrm{kg}$ Feuchtgewicht und einen Mg-Gehalt von $12,7 \mathrm{mmol} / \mathrm{kg}$ Feuchtgewicht aufweisen. Da im Mg-Mangel in einzelnen Tierorganen eine erhöhte Ado- $3^{\prime}: 5^{\prime} P$-Konzentration gefunden wurde $(3,4)$, prüften wir, ob der veränderte Kationen-Gehalt durch unterschiedliche Ado-3': $5^{\prime} P$ Konzentrationen der Zellen verursacht ist. Der Ado$3^{\prime}: 5^{\prime} P$-Gehalt von Yoshida-Zellen, die im normalen Medium vermehrt wurden, beträgt $4,3 \pm 0,16 \mathrm{pmol} / \mathrm{mg}$ Protein, der der Mg-arm gewachsenen Zellen 4,9 $\pm 0,17$ $\mathrm{pmol} / \mathrm{mg}$ Protein. Die Mg-arm gewachsenen Zellen haben damit einen um $14 \%$ höheren Ado-3': $5^{\prime} P$-Gehalt als die Kontrollzellen.

Wenn Ado-3': $5^{\prime} P$ die Permeabilität der Mg-armen Tumorzellen geändert hat, so sollte durch Zusatz von Ado$3^{\prime}: 5^{\prime} P$ die Auswirkung des Mg-Mangels nachgeahmt werden können. Es wurden deshalb verschiedene Konzentrationen von Dibutyryl-Ado-3': $5^{\prime} P$ normalen Tumorzellen zugesetzt und $1 \mathrm{~h}$ später der Gehalt an $\mathrm{Na}^{+}, \mathrm{K}^{+}$, $\mathrm{Ca}$ und $\mathrm{Mg}$ bestimmt. Es zeigte sich (Tab. 2), daß nach Zugabe von 10 bis $100 \mu \mathrm{mol} / 1$ Dibutyryl-Ado- $3^{\prime}: 5^{\prime} P$ die intrazelluläre $\mathrm{K}^{+}$-Konzentration erniedrigt und der $\mathrm{Na}^{+}$und $\mathrm{Ca}-\mathrm{Gehalt}$ erhöht waren. Der Ca-Gehalt war jedoch weniger angestiegen als im Mg-Mangel. Um auszuschließen, daß die intrazellulären Elektrolyte sich durch die wiederholten Waschvorgänge verändern, wurde die Abtrennung der extrazellulären Flüssigkeit von den Zellen durch eine Zentrifugation durch eine Phthalsäuredibutylesterschicht durchgeführt (6). Die Kationenkonzentrationen lagen danach um etwa $10 \%$ höher als die in der Tabelle 2 angegebenen Werte. Die Differenzen nach Dibutyryl-Ado-3': $5^{\prime} P$-Zusatz zu den Kontrollkulturen blieben bestehen.

Tab. 2. Einfluß von Dibutyryl-Ado- $3^{\prime}: 5^{\prime} P$ auf die intrazellulären Konzentrationen von $\mathrm{Na}^{+}, \mathrm{K}^{+}, \mathrm{Ca}$ und $\mathrm{Mg}$ von normalen Tumorzellen

$1 \times 10^{7}$ Zellen $/ \mathrm{ml}$ wurden mit verschiedenen Konzentrationen von Dibutyryl-Ado- $3^{\prime}: 5^{\prime} P 1$ Stunde bei $37^{\circ} \mathrm{C}$ inkubiert, anschließend die intrazellulären Konzentrationen der Kationen bestimmt. Der Variationskoeffizient der Methode beträgt bei $n=14$ Einzelbestimmungen 5,16\%.

\begin{tabular}{|c|c|c|c|c|}
\hline $\begin{array}{l}\text { Konzentration } \\
\text { von Dibutyryl- } \\
\text { Ado- } 3^{\prime}: 5^{\prime} P \\
{[\mu \mathrm{mol} / 1]}\end{array}$ & \multicolumn{2}{|c|}{$\begin{array}{l}\mathrm{Na}^{+} \quad \mathrm{K}^{+} \\
\mathrm{mmol} / \mathrm{kg} \\
\text { Trockengewicht }\end{array}$} & \multicolumn{2}{|c|}{$\begin{array}{l}\mathrm{Ca} \quad \mathrm{Mg} \\
\mathrm{mmol} / \mathrm{kg} \\
\text { Feuchtgewicht }\end{array}$} \\
\hline $\begin{array}{c}100 \\
10 \\
1 \\
0,1 \\
0\end{array}$ & $\begin{array}{l}69,0 \\
64,5 \\
62,4 \\
61,6 \\
60,0\end{array}$ & $\begin{array}{l}280,5 \\
284,3 \\
278,5 \\
287,8 \\
289,5\end{array}$ & $\begin{array}{l}1,91 \\
1,73 \\
1,62 \\
1,62 \\
1,47\end{array}$ & $\begin{array}{l}14,6 \\
14,7 \\
15,8 \\
15,9 \\
15,8\end{array}$ \\
\hline
\end{tabular}

Die Bestimmungen des Ca-Gehaltes der Zellen gestatten jedoch keine Differenzierung zwischen intrazellulärem $\mathrm{Ca}$ und dem $\mathrm{Ca}$, das außen an der Zellmembran gebunden ist. Wahrscheinlich wird im Mg-Mangel infolge der geringeren Kompetition zwischen extrazellulärem $\mathrm{Mg}$ und $\mathrm{Ca}$ an den Liganden der Zellmembran mehr $\mathrm{Ca}$ gebunden als nach Zugabe von Dibutyryl-Ado- $3^{\prime}: 5^{\prime} P$; denn die extrazelluläre Mg-Konzentration ist im Mg-Mangel stark emiedrigt. Die Abnahme des Mg'Gehaltes der Zellen nach Zugabe von Dibutyryl-Ado-3': $5^{\prime} P$ kann durch Efflux von $\mathrm{Mg}$ bzw. durch Verdrängung von intrazellulärem $\mathrm{Mg}$ durch eingeströmtes $\mathrm{Ca}$ bedingt sein.

\section{Diskussion}

In vorhergehenden Untersuchungen (2) hatte sich gezeigt, daß in der Kultur unter Mg-Mangel wachsende Zellen vom 3. Tag an wesentlich langsamer wachsen (Zellzahl am 7. Tag in der Kontrollkultur $8 \times 10^{7}$, in der Mg-arm gewachsenen Kultur $4 \times 10^{7}$ Zellen). Nach 7 Tagen war der intrazelluläre $\mathrm{K}^{+}$-Gehalt von 280 auf $120 \mathrm{mmol} / \mathrm{kg}$ Trockengewicht abgesunken, der $\mathrm{Na}^{+}-$Gehalt von 84 auf $180 \mathrm{mmol} / \mathrm{kg}$ angestiegen. Die Protein-, RNA-, und DNA-Synthese zeigte eine der Wachstumsverlangsamung entsprechende Verminderung. Der Energiestoffwechsel war wenig verändert. Studien mit ${ }^{42} \mathrm{~K}$ hatten ergeben, daß der $\mathrm{K}^{+}$-Efflux zugenommen hatte. Ein signifikanter Unterschied in der Aktivität der Membran-ATPase konnte nicht gefunden werden.

Die hier vorliegenden Ergebnisse zeigen weiterhin, daß im Mg-Mangel der aktive $\mathrm{Na}^{+}$-Transport erhöht ist, der intrazelluläre $\mathrm{Ca}$-Gehalt angestiegen und der $\mathrm{Mg}$-Gehalt abgenommen hat. Die $\left[{ }^{3} \mathrm{H}\right]$ Ouabainbindung ist unverändert. Die intrazelluläre Konzentration von Ado- $3^{\prime}: 5^{\prime} \mathrm{P}$ hat geringfügig zugenommen. Der Aminosäuretransport ist wenig, der Glucosetransport nicht verạnndert.

Es soll diskutiert werden, welche Zusammenhänge zwischen diesen Veränderungen bestehen und wodurch dię im Mg-Mangel auftretenden Veränderungen, wie der verringerte $\mathrm{Na}^{+} / \mathrm{K}^{+}$-Gradient und die Verlangsamung des Zellwachstums, in der Kultur verursacht werden.

Ein verlangsamtes Zellwachstum könnte auf einem veränderten Aminosäuretransport beruhen, der durch die Kopplung von Aminosäure-Transport und $\mathrm{Na}^{+}$Gradient bedingt sein könnte (8). Die geringfügig erniedrigte $\gamma$-Aminobuttersäure-Aufnahme bei den $\mathrm{Mg}$ arm gewachsenen Zellen ist bei den hohen Aminosäurekonzentrationen des Eagle-Spinner-Mediums nicht für die Verringerung des Zellwachstums verantwortlich. Außerdem ist die Kopplung von Aminosäuretransport und $\mathrm{Na}^{+}$-Gradient bei intaktem Energiestoff wechsel gering (8). Die Reduzierung der Proteinsynthese und des Wachstums sind daher wahrscheinlich durch die verringerte intrazelluläre $\mathrm{K}^{+}$-Konzentration bedingt (10). $\mathrm{Ob}$ bei diesen Zellen eine zusätzliche Abnahme von Polyribosomen vorliegt, ist offen. 
Die Abnahme der intrazellulären $\mathrm{K}^{+}$-Konzentration im Mg-Mangel beruht auf einem stark erhöhten passiven $\mathrm{K}^{+}$-Efflux (2). Gleichzeitig besteht ein erhöhter $\mathrm{Na}^{+}$Efflux (aktiver Na-Transport). Daraus folgt, daß im Steady-State auch beim $\mathrm{Na}^{+}$eine verstärkte passive Permeabilität vorliegen muß. Durch das verstärkt einströmende $\mathrm{Na}^{+}$und ausströmende $\mathrm{K}^{+}$kommt es $\mathrm{zu}$ einer veränderten intrazellulären Konzentration von $\mathrm{K}^{+}$und $\mathrm{Na}^{+}$und folglich zu einer Aktivierung der $\mathrm{Na}^{+}-\mathrm{K}^{+}$-Pumpe und so zu einem erhöhten $\mathrm{Na}^{+}$-Efflux. $\mathrm{Da} \mathrm{Na}{ }^{+}$und $\mathrm{K}^{+}$. Transport gekoppelt sind, folgt auch ein erhöhter $\mathrm{K}^{+}$. Transport. Eine Aktivierung der $\mathrm{Na}^{+}-\mathrm{K}^{+}$-Pumpe durch die Veränderung der intrazellulären Kationenkonzentrationen führt offenbar nicht zu einer Veränderung $\operatorname{der}\left[{ }^{3} \mathrm{H}\right]$ Ouabainbindungsgeschwindigkeit. Auch eine Zunahme der Pumpstellen konnte nicht eindeutig nachgewiesen werden.

Als Ursache für die erhöhte passive Permeabilität könnte ein erhöhter Ca-Gehalt der Mg-armen Zellen $(11,12)$ ind/oder ein erhöhter Ado-3':5'P-Gehalt in Frage kommen (9). Ca und Ado-3':5'P haben auch ähnliche Wirkungen, z. B. bei der Aktivierung der Phosphorylase oder Lipase (13-17). Die Zunahme des Ca-Gehaltes kann durch einen vermehrten Ca-Influx (infolge der Abnahme der extrazellulären Mg-Konzentration und einer dadurch verringerten Kompetition mit $\mathrm{Ca}$ ) $\mathrm{zu}$ stande kommen. Durch die Abnahme des membrangebundenen $\mathrm{Mg}$ kann sich auch eine erhöhte passive Permeabilität für Ca auf die gleiche Weise, wie für $\mathrm{K}^{+}$und $\mathrm{Na}^{+}$ergeben. Daneben könnte im Mg-Mangel ein Protein, das für die normale Anreicherung dieser Substanzen nötig ist, nicht mehr in ausreichender Menge gebildet werden (20). Außerdem könnte $\mathrm{Ca}$, das normalerweise in den Mitochondrien angereichert ist, aus diesen in das $\mathrm{Zy}$ toplasma gelangen (18) und dadurch die normale Ca-Verteilung in der Zelle verändern.

Eine Erhöhung des intrazellulären Ca-Gehaltes scheint allerdings nicht immer beim $\mathrm{Mg}$-Mangel-Effekt beteiligt zu sein. So konnte Lehr (19) am Herzmuskel von parathyreoidektomierten Ratten, deren Ca im Serum niedrig war, die typischen $\mathrm{Mg}$-Mangelerscheinungen ohne Erhöhung des zellulären $\mathrm{Ca}-\mathrm{Gehaltes}$ hervorrufen. An $\mathrm{Mg}$-armen $\boldsymbol{E}$. coli, bei denen ebenfalls die typischen Mg-Mangeleffekte beobachtet werden, ist eine Beteiligung des $\mathrm{Ca}$ auszuschließen, da diese Zelle ohne $\mathrm{Ca}$ im Medium gewachsen waren (20).

Die Zunahme des Ado-3':5'P-Gehaltes bei Mg-verarmten Kulturzellen ist sehr gering. Wahrscheinlich hängt die Zunahme des Ado-3':5'P im Mg-Mangel von der sympathischen Innervation des jeweiligen Organs $\mathrm{ab}$. Die in der Mg-Mangel-Kultur erhöhte intrazelluläre Ado- $3^{\prime}: 5^{\prime} P$ Konzentration kann durch eine veränderte Struktur der Zellmembran bzw. Aktivität membrangebundener Enzyme verursacht werden. Der gleichzeitig erhöhte CaGehalt kann die Wirkung des Ado- $3^{\prime}: 5^{\prime} P$ verstärken (17).

Falls die Effekte des Mg-Mangels durch Ado- $3^{\prime}: 5^{\prime} P$ verursacht werden, so muß eine funktionelle Kompartmentierung des Ado- $3^{\prime}: 5^{\prime} P$ in der Zelle angenommen werden (s. hierzu auch 1.c. (21)). Eine derartige Kompartmentierung des Ado- $3^{\prime}: 5^{\prime} P$ wird auch bei der Wirkung des Insulins diskutiert (22). Für eine Wirkung des Ado- $3^{\prime}: 5^{\prime} P$ auf den $\mathrm{K}^{+}-\mathrm{Na}^{+}$-Transport spricht, daß $\mathrm{Zu}$ gabe von Dibutyryl-Ado- $3^{\prime}: 5^{\prime} P$ zu normalen Tumorzellen den Mg-Mangeleffekt imitiert (Tab. 2). Eine Änderung des $\mathrm{Na}^{+}$-Influx nach einem vorhergehenden Anstieg des Ado-3': $5^{\prime} P$ wurde von Sapirstein (23) beobachtet.

Gegen eine generelle Beteiligung des Ado-3':5P an $\mathrm{Mg}$-mangelbedingten Veränderungen spricht aber, daß z. B. an der Schilddrüse im Mg-Mangel keine Veränderungen des Ado-3': $5^{\prime} P$ gefunden wurden, obgleich typische Mangelveränderungen auch in diesem Organ auftreten (24). Es ist daher möglich, daß die durch $\mathrm{Mg}$ Mangel bedingten Effekte je nach Organ oder Versuchsbedingungen verschieden ausgelöst werden. Bei einigen scheint die Zunahme des intrazellulären $\mathrm{Ca}$ die auslösende Ursache zu sein, bei ausgeprägter sympathischer Innervation ist Ado- $3^{\prime}: 5^{\prime} P$ beteiligt.

\section{Literatur}

1. Günther, T. (1970), diese Z. 8, 65-68.

2. Günther, T. \& Averdunk, R. (1970), diese Z. 8, 621-625.

3. Günther, T., Schmalbeck, J. \& Merker, H. J. (1973), dicse Z. $10,425-429$.

4. Günther, T., Schmalbeck, J. \& Merker, H. J. (1973), diese Z. $11,233-236$.

5. Weichselbaum, T. E. (1946), Amer. J. Clin. Path. 16, 40-52.

6. Averdunk, R. \& Lauf, P. K. (1975), Exp. Cell Res. (im Druck).
7. Radkliffe, M. A. (1974), Biochim. Biophys. Acta 339, 303-310.

8. Geck, P., Heinz, E. \& Pfeiffer, B. (1972), Biochim. Biophys. Acta 288, 486-491.

9. Friedmann, N. \& Park, C. R. (1968), Proc. Nat. Acad. Sci. 61, 504-508.

10. Lubin, M. (1967), Nature (London) 213, 451-453.

11. Whittam, R. (1968), Nature (London) 219, 610-612. 
12. Van Rossum, G. D. V. (1970), Nature (London) 225 , 638-639.

13. Rizack, H. (1964), J. Biol. Chem. 239, 392-395.

14. Mansour, T. E. \& Mansour, J. M. (1962), J. Biol. Chem. 237, 629-634.

15. Appelman, M. M., Thomson, W. J. \& Russel, T. R. (1973), Advances in Cyclic Nucleotide Res. 3, 65-98.

16. Rasmussen, H. \& Tenenhouse, A. (1968), Proc. Nat. Acad. Sci. $59,1364-1370$.

17. Ozawa, E. \& Ebashi, S. (1967), J. Biochem. 62, 285-293.

18. Borle, A. B. (1974), Ann. Rev. Physiol. 36, 361-390.

19. Lehr, D. (1973), Myocardiac cell damage, VI. Annual Meeting of the Int. Study Group, Freiburg.
20. Günther, T. \& Mariss, P. (1969), Z. Naturforschung 23b, 334-338.

21. Butcher, R. W., Sneyd, J. G. T., Park, C. R. \& Sutherland, E. W. (1966), J. Biol. Chem. 241, 1651-1653.

22. Walaas, O. \& Walaas, E. (1974), Acta Endocrinol. 191, 93-129.

23. Sapirstein, U. S. \& Scott, W. N. (1973), J. Clin. Invest. 52, 2379-2382.

24. Heaton, F. W. \& Humphray, H. P. (1974), J. Endocrinol. 61, 53-61.

Priv.-Doz. Dr. R. Averdunk Klinikum Steglitz

1000 Berlin 45

Hindenburgdamm 30 